\title{
On-Line Monitoring and Diagnostic System for
}

\section{Turbine-Generator Sets}

\author{
Firas M. Tuaimah, Ph.D \\ University of Baghdad \\ Baghdad, Iraq
}

\author{
F.J.Al-Azawi, Ph.D \\ University of Al-Nahrain \\ Baghdad, Iraq
}

\author{
Mohammed.K.Hadi,Ph.D \\ University of Baghdad \\ Baghdad, Iraq
}

\begin{abstract}
This paper presents a computational methodology to monitor and diagnose electrical faults in large machines, like turbogenerators. To accommodate monitoring requirements, a mathematical model for the turbogenerator is derived based on the magnetic coupled approach. The turbogenerator stator and rotor currents were the guides in fault diagnosis. Digital Signal Processing techniques were applied for current feature detection and recognition. Results obtained show that such approach of condition monitoring initiates necessary alarms to the operator, as the faults develops, which may help in preventing catastrophic failures of the turbogenerators.
\end{abstract}

\section{Keywords}

On-line monitoring, turbogenerator modeling, data base creation, diagnostic.

\section{INTRODUCTION}

Rotating electrical machines play a very important role in the worlds industrial life. In petrochemical and power utilities, the failure of critical rotating machines, such as electric motors and generators is very costly. This is due to loss of production, high emergency maintenance costs and lost revenues. Industry's response towards this problem of unexpected interruptions of work is by using "catch it before it fails" approach. So, the industry started investing heavily on preventive maintenance programs, that is, detecting machine problems before they can result in catastrophic failures [1].

The oldest technique for preventive maintenance was tearing the electrical machine down and then looking at it closely. However, taking the generator out of service is costly and time consuming. This is why today's modern industry management is more interested than ever before in adopting new condition monitoring techniques, on-line or off-line, to assess and evaluate the rotating electrical machines performance condition.

Recent efforts in fault diagnosis include electrical, mechanical, chemical, and magnetic techniques [2].

These techniques are the basis for developing on-line/ or offline rotating electrical machine condition monitoring systems. Electrical and magnetic techniques include magnetic flux measurements, stator current analysis, rotor current analysis, shaft induced voltages, etc. Mechanical techniques include the machine bearing vibration monitoring and, bearing temperature measurement. One of the chemical techniques used is the gas and particulate monitoring in generator hydrogen. Another chemical technique is the analysis of bearing oil.

It is beyond the objective of this paper to cover in detail different monitoring techniques for detecting various faults on large machines. However, one of the electrical techniques, namely generator current signature analysis, will be used to study the effect of any deterioration that may happen in the local electric or magnetic circuit of the turbogenerator.

Several authors have examined the effect of rotor and stator deterioration on machine parameters and performance. Different ways to model and to monitor the machines have been developed. There is a number of published articles on this subject for synchronous and induction machines. In this paper, several related ones will be reviewed.

Mulhaus et al. [3] have described a technique for detecting the presence of rotor shorted turns with the machine on-line depending on the stator current. Their technique relies on the windings of each phase of the generator being divided into two half phases connected in parallel. When a short circuited turn is present on the rotor there is no longer a symmetry between the flux distribution of the two pole faces, and the airgap flux density will contain even harmonics.

Nabil et al. [4] have presented the detection of dynamic air-gap eccentricity in synchronous machines based on modified winding function theory.

G.B.Kliman [5] presented a computer based instrument to detect faults in squirrel cage rotors of induction motors, depending on the line current supply.

The interest in the condition monitoring, on-line/ or off-line, of ac machines has increased tremendously in the last few years because of economic pressures, smaller profit margins and high costs or replacement and spare parts. Therefore, in order to achieve this goal, it is very important to be able to develop simple models for the machines under fault conditions and then analyze the effect of faults on the machine behavior.

In this paper, a mathematical model or a state equation of the machine in its standard form has been developed, which is based on the coupled magnetic circuits approach. This model 
can be used to simulate the machine behavior under healthy and faulty conditions.

The stator current signature patterns of the machine model will be examined that are related to the stator and rotor faults.

\section{SOFTWARE IMPLEMENTATION}

In this paper the monitoring cycle is divided into four parts as shown in Figure 1 below. The first part represents the modeling of the turbogenerator. The second part represents the data base creation or a reference data. The third part defines the recognition criteria, by making some comparison between the received data from the machine and the stored data base (fault detection), and finally the fourth part that represents the output form in which the final decision is given to the operator (diagnostic).

The four parts will be discussed in below.

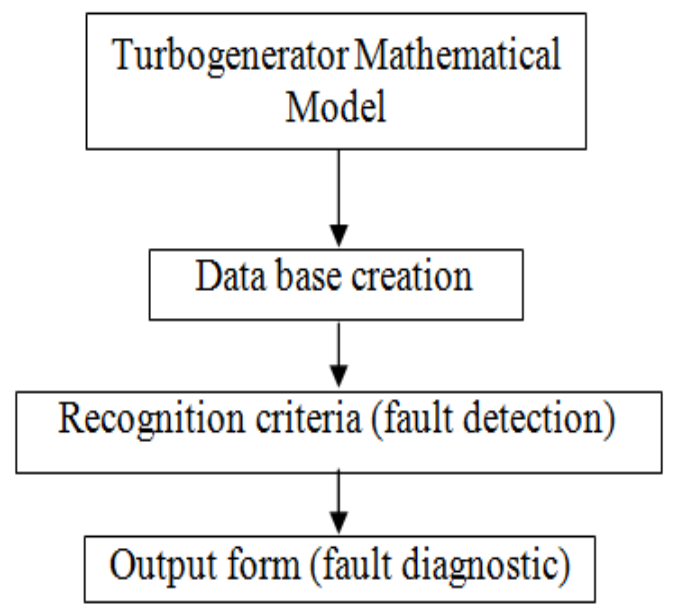

Fig.1 Monitoring cycle algorithm

\section{TURBOGENERATOR MATHEMATICAL MODELING}

Depending on the coupled circuit approach viewpoint the stator and rotor equation sets of a turbogenerator are each expressed in their respective physical circuits [6]. In which the machine will be regarded as a circuit element whose mutual inductances (between stator and rotor) depend on the angular positions of the rotor that runs at synchronous speed.

For the 3-phase winding, the vector of stator and rotor voltages, $\mathrm{V}$, is related to the vector of stator and rotor winding currents, I, and the machine flux linkages, $\lambda$, which will be expressed in terms of the currents and inductances. The result will be a set of nonlinear differential equations describing the dynamic performance of the machine as shown below $[7,8]$ :

$[\mathrm{V}]=[\mathrm{R}][\mathrm{I}]+[\mathrm{P} \lambda]$

Where, $\mathrm{P}=\frac{\mathrm{d}}{\mathrm{dt}}$ (the differential operator)
In terms of the instantaneous winding quantities, the vectors of eq. (1) are:

$$
\mathrm{V}=\left[\begin{array}{c}
V_{a} \\
V_{b} \\
V_{c} \\
V_{f d}
\end{array}\right], \mathrm{I}=\left[\begin{array}{c}
I_{a} \\
I_{b} \\
I_{c} \\
I_{f d}
\end{array}\right], \lambda=\left[\begin{array}{c}
\lambda_{\mathrm{a}} \\
\lambda_{b} \\
\lambda_{\mathrm{c}} \\
\lambda_{\mathrm{fd}}
\end{array}\right]
$$

The matrix of winding resistance has the diagonal form

$$
\mathrm{R}=\left[\begin{array}{cccc}
R_{a} & 0 & 0 & 0 \\
0 & R_{b} & 0 & 0 \\
0 & 0 & R_{c} & 0 \\
0 & 0 & 0 & R_{f d}
\end{array}\right]
$$

In equations (2) \& (3) a, b and c represent the three phase winding of the stator, fd represent the field winding, the damper windings are not seen in the equations because the monitoring system is at steady state.

If $\mathrm{L}$ is a matrix of winding inductances and the inter-winding mutual inductances, then the machine flux linkages in the vector $[\lambda]$, used in eq. (1) may be formed from:

$[\lambda]=[L][I]$

Using the vector $[\lambda]$ from eq. (4) in eq. (1) then gives:

$V]=[R][I]+[P(L I)]$

On expanding the differential of the matrix-vector product (LI):

$$
[\mathrm{V}]=[\mathrm{R}][\mathrm{I}]+\left[\frac{\partial L}{\partial \theta}\right] \cdot \frac{\partial \theta}{\partial t} \cdot[\mathrm{I}]+[\mathrm{L}][\mathrm{PI}]
$$

or

$[\mathrm{V}]=[\mathrm{R}][\mathrm{I}]+[\mathrm{G}] \omega[\mathrm{I}]+[\mathrm{L}][\mathrm{PI}]$

in which

$[\mathrm{G}]=\left[\frac{\partial L}{\partial \theta}\right], \theta=\omega * \mathrm{t}$ and $\omega=\frac{\partial \theta}{\partial t}$

In eq. (8), $\omega$ is the instantaneous angular velocity of rotor relative to the stator.

Since this paper depends on the current in the detection, the rate of change of current for the three phases and the field current can be deduced from equation 7 , as follows:

$[\mathrm{PI}]=[\mathrm{L}]^{-1}[\mathrm{~V}]-[\mathrm{L}]^{-1}[\mathrm{R}][\mathrm{I}]-[\mathrm{L}]^{-1}[\mathrm{G}] \omega[\mathrm{I}](9)$ 
Substituting the inductances and resistances of the machine in the above equation (9) will give:

$$
\begin{aligned}
& {\left[\begin{array}{c}
\frac{\partial \boldsymbol{I}_{a}}{\partial \boldsymbol{t}} \\
\frac{\partial \boldsymbol{I}_{b}}{\partial \boldsymbol{t}} \\
\frac{\partial \boldsymbol{I}_{c}}{\partial \boldsymbol{t}} \\
\frac{\partial \boldsymbol{I}_{f d}}{\partial \boldsymbol{t}}
\end{array}\right]=\left[\begin{array}{llll}
L_{a a} & L_{a b} & L_{a c} & L_{a f} \\
L_{b a} & L_{b b} & L_{b c} & L_{b f} \\
L_{c a} & L_{c b} & L_{c c} & L_{c f} \\
L_{f a} & L_{f b} & L_{f c} & L_{f f}
\end{array}\right]^{-1}\left[\begin{array}{c}
V \operatorname{Sin}(\omega t) \\
V \operatorname{Sin}(\omega t-120) \\
V \operatorname{Sin}(\omega t-240) \\
V \\
f d
\end{array}\right]} \\
& -\left[\begin{array}{cccc}
L_{a a} & L_{a b} & L_{a c} & L_{a f} \\
L_{b a} & L_{b b} & L_{b c} & L_{b f} \\
L_{c a} & L_{c b} & L_{c c} & L_{c f} \\
L_{f a} & L_{f b} & L_{f c} & L_{f f}
\end{array}\right]^{-1} \\
& {\left[\begin{array}{cccc}
R_{a} & 0 & 0 & 0 \\
0 & R_{b} & 0 & 0 \\
0 & 0 & R_{c} & 0 \\
0 & 0 & 0 & R_{f d}
\end{array}\right]\left[\begin{array}{c}
I \operatorname{Sin}(\omega t) \\
I \operatorname{Sin}(\omega t-120) \\
I \operatorname{Sin}(\omega t-240) \\
I_{f d}
\end{array}\right]-} \\
& {\left[\begin{array}{llll}
L_{a a} & L_{a b} & L_{a c} & L_{a f} \\
L_{b a} & L_{b b} & L_{b c} & L_{b f} \\
L_{c a} & L_{c b} & L_{c c} & L_{c f} \\
L_{f a} & L_{f b} & L_{f c} & L_{f f}
\end{array}\right]^{-1} *} \\
& {\left[\begin{array}{cccc}
0 & 0 & 0 & L_{a f d} \operatorname{Sin}(\omega t) \\
0 & 0 & 0 & L_{b f d} \operatorname{Sin}(\omega t-120) \\
0 & 0 & 0 & L_{c f d} \operatorname{Sin}(\omega t-240) \\
L_{\text {afd }} \operatorname{Sin}(\omega t) & L_{b f d} \operatorname{Sin}(\omega t-120) & L_{c f d} \operatorname{Sin}(\omega t-240) & 0
\end{array}\right]} \\
& * \omega *\left[\begin{array}{c}
I \operatorname{Sin}(\omega t) \\
I \operatorname{Sin}(\omega t-120) \\
I \operatorname{Sin}(\omega t-240) \\
I_{f d}
\end{array}\right]
\end{aligned}
$$

This equation means that the rate of change of current in the three phases and the field are dependent on the inductances and resistances of each part. And it will be used, as basis for monitoring since most of electrical faults will be reflected on current as will be seen later.

\section{DATA BASE CREATION}

The mathematical model, which represents the turbogenerator, has been programmed using MATLAB programming language. It can be seen that from equation (10), which represents the model, the rate of change of current is dependent on the inductances and resistances of the stator and rotor portions. Whereas the mutual inductances between the stator and rotor are dependent on the rotor position. On the other hand the mutual inductances between the stator phases, and the self-inductances of the stator phases and rotor are all assumed to have constant values. All these values and parameters (inductances and resistances) will be altered if any type of fault or problem in any level has occurred.

Each fault or problem can be divided into levels, the number of levels can be high or low according to the designer choice and the amount of accuracy wanted.

Seven levels are chosen, in thus work according to the degradation in the selected problem.

Type of faults and problems handled in this paper are:

a. Short circuit in the stator windings

b. Overtemperature in Phases a, b and c.

c. Volts-Per-Hertz (V/Hz) Monitoring

d. Underfrequency and Overfrequency

e. Short Circuit and Open Circuit in Field Winding

f. Overtemperature in the Field Winding

Taking the short circuit in phase a as example

If severe mechanical movement of the windings occur during its daily operation, then winding subconductors insulation may fail due to this movement leading to arcing because of subconductor shorting, which in worst cases has eroded and melted other subconductors. If this happens near other earthed metalwork then an earth fault can occur and sometimes serious phase-to-phase fault may happen. The progression of faults may produce very high temperatures and since the insulation between the windings is the weakest components of any electrical machine then the isolated material may be defected leading to serious increase in discharge activity. An increase of temperature due to subconductor shorting in the windings, above the design limits may lead to lamination defects and then the stator core defect may happen, which is a rare event.

The value of the impedance can be taken as a measure to determine the severity of the fault, this impedance in turn will produce changes in the phase current. 
The seven levels chosen, which indicate the severity of the fault is given in table 1

Table.1 Short circuit severity levels for phases $a, b \&$.

\begin{tabular}{|c|c|}
\hline Levels & Impedance (p.u) \\
\hline Healthy & 1 \\
\hline Alarm 1 & 0.95 \\
\hline Alarm 2 & 0.9 \\
\hline Alarm 3 & 0.85 \\
\hline Alarm 4 & 0.8 \\
\hline Alarm 5 & 0.7 \\
\hline Faulty & 0.6 \\
\hline
\end{tabular}

In order to finish the data base creation process for this type of fault (short circuit), the above levels that show the fault severity are used in the turbogenerator mathematical model of equation (10) to get the desired current signals for each level as given in Figure 2.

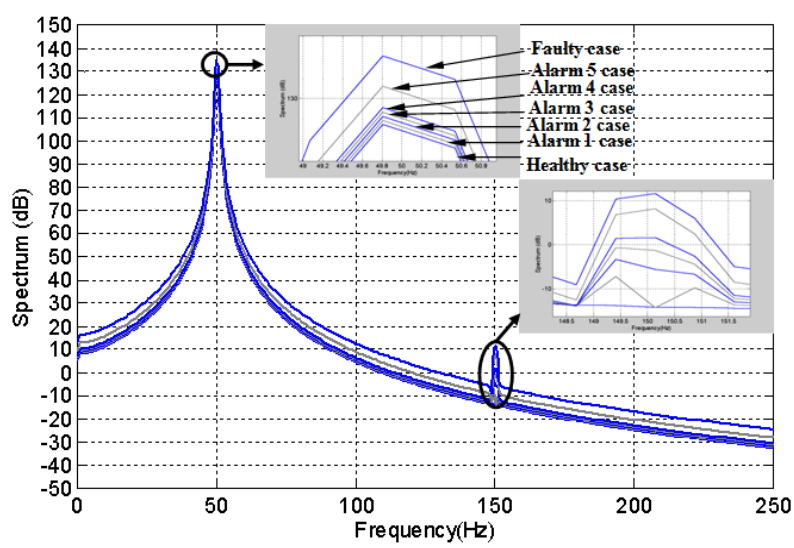

Fig. 2: Spectrums showing the short circuit levels in phase a

For each level, 2049 samples from frequency spectrum of the associated time signal are added to the database. This process will continue until the creation process for this type of fault is finished for the seven levels.

The same process will be continued for all the selected faults and problems, in order to finish the database creation.

\section{RECOGNITION CRITERIA (FAULT DETECTION)}

Recognition between the received data from the machine and the stored data can be divided into two classes:

1) Objective criteria.

2) Subjective criteria.

The objective criteria are borrowed from digital signal processing and information theory and provide us with equations that can be used to measure the error between the stored data and the received data from the machine. Subjective criteria require the definition of a qualitative scale to assess the received case. This scale can then be used by human test to determine the case type, by monitoring the graphs to make the recognition. The objective criteria, is widely used, because of its accuracy.

There are a number of objective methods that compute the distance or error between number of cases, which they are: Euclidean distance method (root-mean-square error $\mathrm{e}_{\mathrm{RMS}}$ ), Standardized Euclidean distance method, Mahalanobis distance method, City Block method, and Minkowski method. These methods differ from each other according to their use, since they deal with different fields of study.

The objective measure that is adopted in this work, is the Euclidean distance method (root-mean-square error $\mathrm{e}_{\mathrm{RMS}}$ ). This method gives us accurate results as will be shown later.

The distance or error between an original (stored) case samples and the received case samples can be defined as:

Error $(\mathrm{s})=\hat{\imath}(\mathrm{s})-\mathrm{I}(\mathrm{s})$

Where $\mathrm{I}(\mathrm{s})=$ the original stored data

$$
\hat{\imath}(\mathrm{s})=\text { the received data }
$$

Next, we can define the total error for $\mathrm{N}$ samples as:

$$
\text { Total error }=\sum_{s=0}^{N-1}[\hat{\mathbf{1}}(\mathrm{s})-\mathrm{I}(\mathrm{s})]
$$

The root-mean-square error is found by taking the square root of the error squared divided by the total number of samples:

$$
\mathbf{e}_{\mathbf{R M S}}=\sqrt{\frac{1}{N^{2}} \sum_{s=0}^{N-1}[\hat{\mathbf{1}}(\mathrm{s})-\mathrm{I}(\mathrm{s})]^{2}}
$$

For example, if the received data $\mathrm{Xr}=\left[\begin{array}{lll}1 & 2 & 3\end{array}\right]$ for three samples

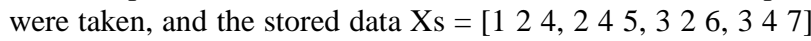
or $\mathrm{Xs}=\left[\mathrm{X}_{\mathrm{s} 1}, \mathrm{X}_{\mathrm{s} 2}, \mathrm{X}_{\mathrm{s} 3}, \mathrm{X}_{\mathrm{s} 4}\right]$, and applying the above equation, then the total root-mean-square error will be as shown in graph below, which shows that the received data is nearest to the first stored data, as given in Figure 3.

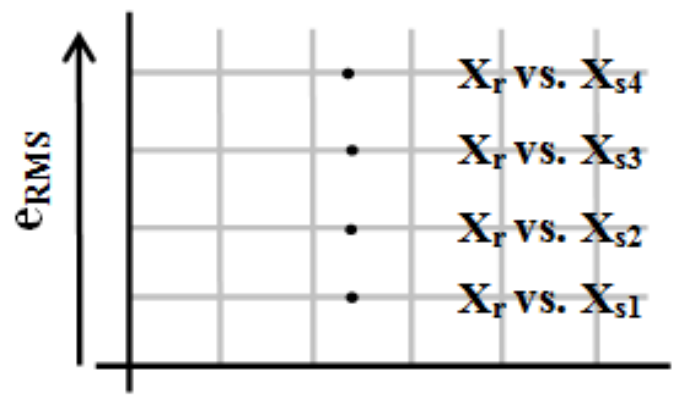

Fig. 3: The error or distance between the received signal and number of stored signals. 


\section{MONITORING RESULTS OUTPUT FORM}

After completing the process of recognition for the selected problem (as given above), the results should be displayed in a simple form for the operator or the engineer who is working on that plant, as shown in Figure 4.

The results given to the operator will be in the form of instructions and comments, which depends on the fault type and its severity. These instructions are taken from the past history for the same type of machine, from the operators experience and from the machine design.

Visual Basic 6 programming language was used to build that form as shown in Figure 4 Which consists of the following:

a. All the faults and problems handled in this work, with the seven selected levels for each.

b. Alarm trouble shouting.

c. History of case signals.

d. Useful notes about the machine (text form).

e. Help about dealing with all windows (text form).

f. Some important Figures about some locations in the machine.

g. Phases a, b, c models and field model.

h. Current date and time.

i. Window showing the signal interpretation.

j. Window showing the used abbreviations (text form).

k. Window showing the turbogenerator electrical and mechanical description.

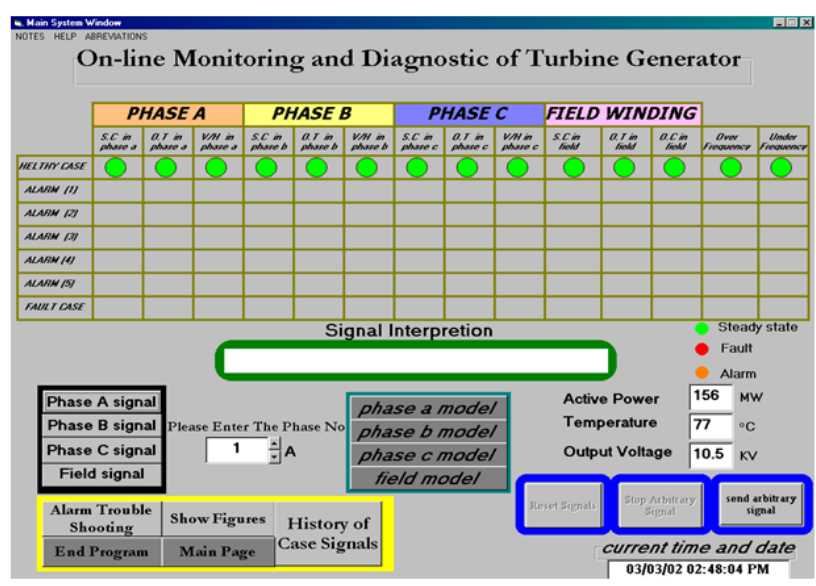

Fig. 4: Monitoring system main menu.

\section{THE DIAGNOSTIC TASK}

It is the task that closes the loop in the monitoring activity by virtue of the fact that decisions made here should influence operational and maintenance strategy. It is useful to stress on the fact that the commonest form of diagnostic processor is still the man. It is true that expert systems derived from knowledge bases are beginning to appear in diagnostic procedures, but the final arbiter remains the man.

The quantity of information given to the operator should be suitable to the case. To simplify, it is possible to identify two situations which have two opposite causes: sometimes the supervision operator can be saturated by an information, overload, whereas some other times an information underload, can deteriorate the operators mental model of the supervised process. These situations are normally due to the operators tendency to wait for the alarm to act, instead of trying to foresee or anticipate abnormal process states. Since human operator has to face with an alarm cascade, it becomes difficult to avoid or manage interpretation and/ or action failures. Moreover, there are some aid supports which minimize operators attention and so his anticipate capabilities. To avoid these perverse effects and to make operators work more active, future supervision and monitoring has to be human centered, that it has to take into account and integrate cognitive capabilities of human operator such as perception, anticipation and reflection capabilities. Our objective is to provide the operator with the sufficient degree of information to realize the different tasks required during the monitoring activity.

\section{CONCLUSIONS}

The main conclusions from the work are:

a. This type of monitoring systems, which depends on the current in fault diagnosis, does not need any type of sensors to be connected inside or outside the machine except the current transformer (sensorless method). And can be used to detect number of faults as well as number of abnormal problems like the overtemperature, overfrequency, underfrequency and etc.

b. The whole spectrum is taken in the comparison, which begins from 0 to $250 \mathrm{~Hz}$ with $500 \mathrm{~Hz}$ sampling frequency, and sampled to 2049 sample. The reason for taking the whole spectrum is that, these spectrums are not identical in features, because we deal with a number of faults and problems. In faults like short circuit or open circuit number of harmonics appear ( $1^{\text {st }}$ harmonic, $3^{\text {rd }}$ harmonic), whereas in the abnormal problems like the overtemperature, overfrequency, underfrequency, $\mathrm{V} / \mathrm{Hz}$, the first harmonic only seen. Besides the spectrum level is shifted according to the type of fault or problem and according to severity level.

c. Implementing the on-line monitoring systems with its diagnostics capabilities on the turbogenerators reduces the shut down times

d. The proposed monitoring system can be used as a teaching program for the junior engineers who are working in the field of diagnostics and repairing the parts of the machine.

e. This monitoring system is generic and can be applied to another rating and types of turbogenerators by making some change on the database.

f. The proposed monitoring system perpetuates the past experience of the old operators on the same type of machines. This can be done by saving these experiences on 
the same monitoring system computer, and producing them to the new operators or engineers in case of emergency.

\section{REFERENCES}

[1] P.J.Tavner, B.G.Gaydon, and D.M.Ward: Monitoring generators and large motors, IEE Proceedings, Vol.133, Pt.B.No.3, May 1986. [2] Peter J.Tavner and James Penman: Condition monitoring of electrical machines, John Wiley \& Sons, 1987.

[3] Muhlhaus, J., Ward, D.M. and Lodge, I.: The detection of shorted turns in generator rotor windings by measurement of circulating stator currents, Proc. of Int. Conf. On Electrical Machines-Design and applications, IEE, London, Conference Publn. 254, September 1985, pp 100103.

[4] Nabil A.A., Hamid A.T.: A Novel Method for Modeling Dynamic Air Gap Eccentricity in Synchronous Machines Based on Modified Winding Function Theory, IEEE
Trans. Energy Conversion, Vol.13, pp.156-162, June 1998.

[5] A.E.Fitzgerald, Charles Kingsley, JR., Alexander Kusko: Electric Machinery, McGraw-Hill Kogakusha, 1971.

[6] F.J.AL-Azzawi: A training course on stability of electrical power systems, Amman-Jordan, August 1996.

[7] D.O'kelly and Simmons: Introduction to generalized electrical machine theory, 1968.

[8] C.Emmanouilidis, Dr.J.Maclntyre, Prof. C. Cox.: Neurofuzzy computing aided machine fault diagnosis. Proc. of JCIS'98, The fourth joint conference on information sciences. Research Triangle Park, North Carolina, USA, Vol.1.pp.027-210.1998

[9] Scott E Umbaugh: Computer Vision and Image Processing: a practical approach using CVIP tools, Prentice Hall 1998.

[10] Statistics toolbox for use with MATLAB, version 2 , Mathworks, March 1996. 